\title{
Multilevel and Local Time-stepping Discontinuous Galerkin Methods for Magma Dynamics
}

\author{
S. Tirupathi - J. S. Hesthaven - Y. Liang - M. Parmentier
}

Received: date / Accepted: date

\begin{abstract}
Discontinuous Galerkin (DG) method is presented for numerical modeling of melt migration in a chemically reactive and viscously deforming upwelling mantle column at local chemical equilibrium. DG methods for both advection and elliptic equations provide a robust and efficient solution to the problems of melt migration in the asthenospheric upper mantle. Assembling and solving the elliptic equation is the major bottleneck in these computations. To address this issue, adaptive mesh refinement and local time-stepping methods have been proposed to improve the computational wall time. The robustness of DG methods is demonstrated through two benchmark problems by modeling detailed structure of high-porosity dissolution channels and compaction dissolution waves.
\end{abstract}

Keywords Numerical solutions · Discontinuous Galerkin - Adaptive mesh refinement - Local timestepping · Mid-ocean ridge processes · Magma migration $\cdot$ Physics of magma bodies

\footnotetext{
S. Tirupathi

Division of Applied Mathematics, Brown University, Providence, USA

E-mail: seshutir@ie.ibm.com

Present address: IBM Research - Ireland, Damastown Industrial Park, Dublin, Ireland

J. S. Hesthaven

Mathematics Institute of Computational Science and Engineering,EPFL, Lausanne, Switzerland

Y. Liang

Department of Earth, Environmental and Planetary Sciences, Brown University, Providence, USA

M. Parmentier

Department of Earth, Environmental and Planetary Sciences, Brown University, Providence, USA
}

\section{Introduction}

Magma dynamics deals primarily with the physics of magma generation and migration in the Earth and planetary interior and is a subject of extensive geophysical fluid dynamics investigations. The governing partial differential equations for conservation of mass, momentum and energy to model magma dynamics are highly nonlinear $[4,30,37]$ and have to be solved numerically in almost all geological applications. Finite difference, finite volume (FVM) and finite element methods (FEM) are the standard spatial discretizations to solve the mass conservation or hyperbolic equations arising in magma dynamics. Early numerical study of Spiegelman and coworkers [40] used low order finite difference methods. While the spatial discretization of these problems was very efficient and simple, these methods cannot be easily extended to high-order methods on general grids due to extended stencils required for reconstruction [21]. Finite volume methods have been used to model melt migration beneath the mid-ocean ridge [15, 23], and these methods provide the aforementioned geometric flexibility for lower order. However, to increase the order of accuracy in more than one dimension requires costly reconstruction. Unlike finite difference and finite volume methods, finite element discretization provides a very good alternative to address accuracy issues as these methods assume that the solution is a linear combination of the basis functions which can be made arbitrarily high order [26]. The geometric flexibility of FEM also makes it really useful to implement in melt migration problems and commercially available software packages make application of FEM to magma dynamics straightforward [5]. While these methods are ideal for elliptic equations, to solve the pressure equation in magma dynamics, they have some drawbacks 
for transport equations. Globally defined basis functions and the symmetric structure of the basis functions makes the semi-discrete scheme costly to implement and causes stability issues respectively. Apart from the standard methods, hybrid schemes have been developed to study melt migration problems such as semiLagrangian algorithms [38], Mixed Fourier CollocationDiscontinuous Galerkin Method [35] and mixed discontinuous Galerkin and high-order finite difference method [28]. However, these methods require significant amount of coding and can be prohibitively costly when extended to three dimensions.

The requirements of high order accuracy with geometric flexibility through local approximation like finite elements with stability requirements satisfied through methods like FVM for transport problems can be achieved through discontinuous Galerkin (DG) methods. DG methods are a class of finite element methods which use completely discontinuous basis functions. DG method was first proposed to solve the steady-state neutron transport equation by [31]. The rigorous mathematical framework of DG methods for time dependent problems was carried out by Cockburn and coworkers in a series of papers ([8] - [12]). There are several advantages of the DG methods making them attractive for applications of the type considered here. These include their ability for easy handling of complicated geometry and boundary conditions (an advantage shared by FEM), their flexibility for easy h-p adaptivity (splitting elements in space or increasing the polynomial degree) including changes of approximation orders between neighboring elements and allowing general meshes with hanging nodes, their compactness (evolution of an element depends only on information from immediate neighbors) and hence efficient parallel implementation. These properties have led to a rapid spread of diverse applications to a wide range of computational problems such as aeroacoustics [32], electro-magnetism [22], gas dynamics [45], modeling of shallow water [16], reactive transport in porous media [42] among many others.

DG methods are a combination of FEM and FVM with the mesh generated into elements like FEM but the solution can be discontinuous between elements. Consequently the mass matrix for the transport equations are local rather than global and hence can be easily inverted. Stability of DG methods is ensured with suitable flux choices like FVM. High order methods can be easily generated as well through local element based basis required for DG methods while ensuring stability. While DG methods satisfy all the requirements for high order methods, it also results in an increase in the total number of degrees of freedom. Also, the advantages of DG methods do not extend to elliptic problems where the locality of basis functions and flux choices is not as important. On the other hand, the elliptic operator with DG methods tends to be more sparse than the corresponding FEM method. Further reference and detailed discussions on DG methods for advection and elliptic equations can be found in $[2,8,21,42]$.

The main objective of the present study is to develop an efficient and accurate numerical model using DG methods that can be used to study the interaction between the melt and solid during melt migration in a viscously deforming, partially molten and chemically reactive mantle column. The governing equations track the evolution of the soluble mineral (orthopyroxene or opx for short), porosity and pressure in an upwelling heterogeneous mantle. While this paper concentrates on the numerical method and so all the examples considered in this paper are in 2D for simplicity, the numerical method is general enough to be extended to 3D. However, to expand to three dimensional space, it would require significant amount of coding even if the method is the same at the algorithmic level. Hence, use of existing libraries makes it convenient to extend these results to $3 \mathrm{D}$ and in this paper we use the software library called deal.II (Differential Equations Analysis Library) which gives us the flexibility of using a united interface to write programs in a dimension independent way and take advantage of the generic functions required for discontinuous Galerkin methods that would otherwise have to be coded explicitly [3]. As described in [35] we explicitly track the evolution of the soluble mineral (opx) to understand the formation of the observed opx-depletions and their spatial and temporal relation to localized melt flow in regions of high permeability. We evolve the governing system by solving the pressure equation using the current porosity values, and then explicitly evolve porosity and opx by a time step. While this method works perfectly to study the characteristics of melt migration, there is a significant bottleneck in solving these problems. The time required to assemble and solve the pressure equation significantly slows down the computational time required to capture the characteristics of high-porosity channel formation during melt migration with a globally refined mesh. Since the region of interest is a small fraction of the whole computational domain, adaptive mesh refinement provides a very good alternative to efficiently resolve the essential flow features of the system. In addition to adaptive mesh refinement, since the pressure equation weakly affects the advection equations in the channel problem, local time-stepping can be employed to significantly improve the computational time.

The remaining of this paper is organized as follows. In Section 2, we outline the governing equations. In Sec- 
tion 3, we present the numerical discretization consisting of a discontinuous Galerkin scheme for the given set of equations. DG methods are checked for robustness in handling magma dynamics problems through convergence and scalability analysis. In Section 4, we discuss benchmark cases involving high-porosity channels and compaction-dissolution waves using DG methods. We first consider high-porosity channels, understanding the bottleneck in solving magma dynamics equations and strategies that can be employed to resolve this bottleneck using local time-stepping methods. Next, we show the same results as case one but with adaptive mesh refinement with the assumption that the location of channel formation is not known apriori and thus underscoring the importance of adaptive methods for realistic problems. In the third case of compaction-dissolution wave problem, the performance of DG methods and the importance of parallelization is emphasized as the problem size is considerably bigger than channel problems. These methods are further improved by considering anisotropic refinement.

\section{Physical Model}

We consider a three-phase system with an interconnected melt or fluid phase and a solid that consists of an insoluble mineral, olivine (ol), and a soluble mineral, opx. We are interested in the spatial and temporal variations in the volume fraction of melt $\left(\phi_{f}\right)$, soluble mineral opx $\left(\phi_{o p x}\right)$, and the pressure difference between the melt and the solid matrix or effective pressure $p$ in this system. The governing equations for our model system have been previously discussed in $[1,40]$, and later extended in $[19,35,36]$ to incorporate solid upwelling, porosity-dependent bulk viscosity, and explicit tracking of the soluble mineral. A reactive time scale was used in [19], whereas an upwelling time scale was used in [36]. Below we present the non-dimensionalized equations on an upwelling time scale (see Appendix for details of the governing equations and their non-dimensionalization). The evolution of the melt fraction, $\phi_{f}$, and opx abundance, $\phi_{o p x}$, are given by the mass conservation equations. In non-dimensional form, we have

$\frac{\partial \phi_{f}}{\partial t}=-\frac{\partial \phi_{f}}{\partial z}+\phi_{f} p+\delta \Gamma_{o p x}$

$\frac{\partial \phi_{o p x}}{\partial t}=-\frac{\partial \phi_{o p x}}{\partial z}-\frac{\phi_{f}^{0}}{\phi_{o p x}^{0}} \frac{\delta}{(1-\beta)} \Gamma_{o p x}$,

where time $t$ is scaled to solid upwelling time, spatial coordinates are scaled by compaction length, and volume fractions of melt and opx are scaled to reference or background values of $\phi_{f}^{0}$ and $\phi_{o p x}^{0}$ at the inflow. The other symbols denoted in the equations for porosity and opx are the normalized solubility gradient $(\delta)$, ratio of melt to solid velocity $(R)$, initial melt fraction $\left(\phi_{f}^{0}\right)$ and ratio of opx dissolution rate to olivine precipitation rate $(\beta)$. Darcy's law yields the fluid velocity $\mathbf{v}$ relative to the uniform solid upwelling velocity via the equation

$\phi_{f} \mathbf{v}=R^{-1} \phi_{f} \mathbf{n}_{z}-\phi_{f}^{3}\left(R^{-1} \nabla p-\mathbf{n}_{z}\right)$

where $\mathbf{n}_{z}$ is the unit vector in the vertical dimension. Coupling Darcy's law to the momentum equation for solid matrix, the following elliptic equation is derived for the fluid-solid pressure difference $p[36]$,

$-\nabla \cdot\left[\phi_{f}^{3} \nabla p\right]+\phi_{f} p=-R \frac{\partial \phi_{f}^{3}}{\partial z}$.

Finally, under the assumption of local chemical equilibrium, the dissolution rate, $\Gamma_{o p x}$, is proportional to the Darcy flux, $\phi_{f} \mathbf{v}$, and is computed algebraically as

$\Gamma_{o p x}=\frac{\phi_{f}\left(\mathbf{v} \cdot \mathbf{n}_{z}\right)}{1-\delta z} \mathcal{I}_{o p x}$

where the indicator function $\mathcal{I}_{o p x}$ returns 1 if $\phi_{o p x}$ is positive and zero otherwise. In this way, dissolution occurs only if the soluble mineral opx is present. The three right-hand-side terms of Eq. 1 represent solid upwelling, compaction, and dissolution, respectively. Collectively the three processes effectively give rise to an advectionreaction type equation. Eq. 1 contains the dimensionless parameter $\delta$, representing the solubility gradient of the equilibrium concentration of the soluble mineral in the fluid. Here the coefficient of $\Gamma_{o p x}$ is the opx dissolution rate, representing the rate at which opx is consumed in the dissolution process. Hence, the dissolution term in Eq. 2 carries an opposite sign as in Eq. 1; the former represents depletion of opx whereas the latter represents generation of melt.

\subsection{Parameter Values, Initial and Boundary Conditions}

Linear stability analysis and numerical simulations of the full nonlinear problem described by Eqs. 1 - 5 revealed presence of three dynamic regimes: (1) an unstable fingering regime where small perturbations in porosity grows monotonically; (2) an unstable wave regime where small perturbations in porosity grows periodically; and (3) a stable regime where infinitesimal perturbation in porosity decays exponentially $[19,28,36]$. For melt migration beneath the mid-ocean ridge spreading centers, stable and wave regimes may be more relevant $[28,36]$. Simulations of high-porosity channels in 
$[27,36]$ were conducted in the stable regime with a prescribed time independent boundary condition for porosity at the inflow. In this study, we consider benchmark problems of stable high-porosity channel formation and unstable compaction-dissolution wave formation in Section 4. For channel problems, we follow [36] and take the boundary condition at the inflow $(z=0)$ to be a Gaussian pulse added to unity,

$\phi_{f}(x ; 0 ; t)=1+A_{1} \exp \left(-100\left(x-\frac{x_{\max }}{2}\right)^{2}\right)$,

where $A_{1}$ is a non-zero constant and $x_{\max }$ is the domain width. For an initial condition we extend Eq. 6 across the entire vertical column, although results presented in this study are independent of the initial conditions except the early time $\left(t<z_{\max }\right)$, where $z_{\max }$ is the domain height. The initial condition for $\phi_{o p x}$ varies linearly in the $\mathrm{z}$ direction based on the intuition obtained from the steady-state solution in the equivalent $1 \mathrm{D}$ problem. We assume periodicity in the $\mathrm{x}$ dimension. For the pressure equation, the boundary conditions are periodic in $\mathrm{x}$ and $\mathrm{y}$. Also from [19], we have the boundary conditions at $z=0$ and $z=z_{\max }$,

$p(x, 0, t)=-\frac{\delta(1+R)}{1+(n R)^{-1}}$

$\frac{\partial p\left(x, z_{\max }, t\right)}{\partial z}=0$.

As in [36], we set $R=100$ and $\delta=10^{-2}$ in the simulations presented in this study. In the wave regime, we consider random initial and boundary conditions for porosity while the $\phi_{o p x}$ initial and boundary conditions remain the same. The input parameters used in this paper are listed in Table 1.

\section{Numerical discretization}

\subsection{Numerical Schemes}

To solve the system Eqs. 1 - 5 we employ a discontinuous Galerkin (DG) scheme for the hyperbolic equations (Eqs. 1, 2) and interior penalty discontinuous Galerkin (IPDG) method for the elliptic equation (Eq. 4). The rationale behind these choices is as follows. For the advection equations, DG methods are well suited for conservation laws and are robust and fast due to locality. IPDG methods were used for the elliptic equation as they are easily parallelizable and require very little storage compared to other DG methods like local discontinuous Galerkin method. Complete details of the implementation of this method are given in [33]. Proceeding by a method-of-lines approach, the spatial operations in the hyperbolic equations (Eqs. 1 - 2) are discretized to produce a system of differential-algebraic equations that are a function of time only and can be integrated with a 3rd-order Runge-Kutta method.

\subsection{Full Numerical Method}

The domain $\Omega$ is triangulated into K non-overlapping elements such that

$\Omega \simeq \Omega_{h}=\bigcup_{k=1}^{K} D^{k}$.

The function space $V_{h}$ is defined as element wise discontinuous polynomials of degree $N$ such that

$V_{h}=\left\{v_{h} \in L^{2}(\Omega):\left.v_{h}\right|_{D^{k}} \in P^{N}\left(D^{k}\right), k=1,2 \ldots K\right\}$,

where $v_{h}$ is the multidimensional Legendre polynomial in the element $k, L^{2}$ is the space of square integrable functions and $P$ is the polynomials space with $\mathrm{N}$ as the degree of the polynomial chosen according to the required order of accuracy. We look for solutions $\phi_{f_{h}}$ , $\phi_{o p x_{h}}$ in this function space. Following the procedure in [21], the discretized form of the porosity evolution equation (Eq. 1) is

$$
\begin{array}{r}
\int_{D^{k}} v_{h} \partial_{t} \phi_{f_{h}}^{k} d \mathbf{x}=\int_{D^{k}} \phi_{f_{h}}^{k} \mathbf{n}_{z} \cdot \nabla v_{h} d \mathbf{x}+ \\
-\oint_{\partial D^{k}} v_{h}\left(\phi_{f_{h}}^{k}\right)^{*} \mathbf{n}_{e} \cdot \mathbf{n}_{z} d \mathbf{x}+\int_{D^{k}} v_{h} p \phi_{f_{h}}^{k} d \mathbf{x}+ \\
\int_{D^{k}} \delta v_{h} \Gamma_{o p x_{h}}^{k} d \mathbf{x},
\end{array}
$$

where $\mathbf{n}_{e}$ is the outward pointing normal to the cell face $\partial D^{k}$. We choose an upwinding flux as

$$
\begin{aligned}
\phi_{f}^{k, *}\left(\phi_{f}^{k,-}, \phi_{f}^{k,+}\right) & =\phi_{f}^{k,-}, \mathbf{n}_{z} \cdot \mathbf{n}_{e} \geq 0 \\
& =\phi_{f}^{k,+}, \mathbf{n}_{z} \cdot \mathbf{n}_{e}<0
\end{aligned}
$$

for the cell face $\partial D^{k}$. The quantities $\phi_{f}^{k,+}$ correspond to the face values of the current cell on the given face and $\phi_{f}^{k,-}$ are the values on the face taken from the neighboring cell. The opx evolution equation is discretized in a similar fashion

$$
\begin{array}{r}
\int_{D^{k}} v_{h} \partial_{t} \phi_{o p x_{h}}^{k} d \mathbf{x}=\int_{D^{k}} \phi_{o p x_{h}}^{k} \mathbf{n}_{z} \cdot \nabla v_{h} d \mathbf{x}+ \\
-\oint_{\partial D^{k}} v_{h}\left(\phi_{o p x_{h}}^{k}\right)^{*} \mathbf{n}_{e} \cdot \mathbf{n}_{z} d \mathbf{x}- \\
\int_{D^{k}} \frac{\phi_{f}^{0}}{\phi_{o p x}^{0}} \frac{\delta}{(1-\beta)} v_{h} \Gamma_{o p x_{h}}^{k} d \mathbf{x} .
\end{array}
$$

Lastly, the elliptic equation is a modified Helmholtz equation and this is discretized using IPDG Method. 
Table 1 Values of model parameters used in simulations

\begin{tabular}{|c|c|c|c|}
\hline Parameter & Symbol & Values for channel problem & Values for wave problem \\
\hline \hline Normalized solubility gradient & $\delta$ & 0.01 & 0.01 \\
\hline Ratio of melt to solid reference velocities & $R$ & 100 & 100 \\
\hline Initial melt fraction & $\phi_{f}^{0}$ & 0.02 & 0.01 \\
\hline Initial opx fraction & $\phi_{o p x}^{0}$ & 0.15 & .20 \\
\hline Ratio of opx dissolution rate to olivine precipitation rate & $\beta$ & 0.5 & 0.5 \\
\hline Porosity exponent in permeability & $n$ & 3 & 3 \\
\hline Non-dimensional domain length in x direction & $x_{\max }$ & 1.0 & 5.7 \\
\hline Non-dimensional domain length in z direction & $z_{\max }$ & 2.0 & 6.8 \\
\hline
\end{tabular}

Referring [2,42], discretization for the pressure equation becomes,

$$
\begin{array}{r}
\sum_{k=1}^{K}\left(\int_{D^{k}} \phi_{f}^{3} \nabla p \cdot \nabla v+\int_{D^{k}} \phi_{f} p v\right)+ \\
\epsilon \sum_{e \in \partial D_{i n} \cup \partial \Omega_{d}} \int_{e}\left\{\phi_{f}^{3} \nabla v \cdot \mathbf{n}_{e}\right\}[p] \\
-\sum_{e \in \partial D_{i n} \cup \partial \Omega_{d}} \int_{e}\left\{\phi_{f}^{3} \nabla p \cdot \mathbf{n}_{e}\right\}[v] \\
+\sigma \sum_{e \in \partial D_{i n} \cup \partial \Omega_{d}} \int_{e}[p][v] \\
=\sum_{k=1}^{K} \int_{D^{k}}-R \frac{\partial \phi_{f}^{3}}{\partial z} v+\sum_{e \in \partial \Omega_{d}} \int_{e}\left(\epsilon \phi_{f}^{3} \nabla v \cdot \mathbf{n}_{e}+\sigma v\right) g_{D} .
\end{array}
$$

The discretized form of the pressure equation given in 14 has notations that have special meanings. The jump terms required for stability, correspond to variables in square brackets and averages of variables across faces are denoted in flower brackets. These values are calculated for every edge, e, belonging to the family of internal edges, $\partial D_{i n}$ and edges along the boundary, $\partial \Omega_{d}$ of the domain. The choice of the value of the parameter, $\epsilon$, results in different schemes with respective advantages and disadvantages $[2,42] . \sigma$ is called the penalty parameter that penalizes the jump in the function values across the cell face. As a solver, we use a matrixfree implementation of Biconjugate gradient stabilized (BiCGStab) iterative method with a block Jacobi preconditioner for the elliptic equation [34].

The basic DG formulation suffices to solve the governing set of equations when there are no steep gradients in the time variation of porosity which is generally true in the channel problem. But in the wave regime, the random variation of porosity combined with the source term, $\Gamma_{o p x}$, which scales with $z_{\max }$ results in numerical oscillations. These oscillations can be controlled by adding an artificial viscosity. But the additional viscosity term that is introduced must primarily act in regions of numerical oscillations and be small in regions where the porosity variation is smooth. We consider the entropy viscosity proposed by [46], which is based on the residual of the governing equations. The artificial viscosity is a piecewise constant function defined by:

$\left.\nu\right|_{k}=\alpha \min \left\{c_{\max } h_{k}, c_{E} h_{k}^{2} \max \left(D_{1}\left(\phi_{h}\right)\right)\right\}$,

where $\alpha$ is a stabilization constant that is tweaked to scale the artificial viscosity according to the requirements of the problem. The second term $D_{1}\left(\phi_{h}\right)$ is further defined as:

$D_{1}\left(\phi_{h}\right)=\max \left(\max _{x \in k}\left|R e_{i}\left(\phi_{h}\right)\right|, \max _{x \in \partial k}\left|J_{i}\left(\phi_{h}\right)\right|\right) / N_{h}$.

The individual terms in the definition of $D_{1}\left(\phi_{h}\right)$ are given by:

$R e_{i}\left(\phi_{h}\right)=\frac{\phi_{f}^{n}-\phi_{f}^{n-1}}{\Delta t^{n}}+\partial_{z} \phi_{f}^{(a)}-p^{(a)} \phi_{f}^{(a)}-\Gamma_{o p x}^{(a)}$,

$J_{i}\left(\phi_{h}\right)=\left[\phi_{f}^{(a)}\right]$

$N_{h}\left(\phi_{h}\right)=\operatorname{var}\left(\phi_{f}^{n+1}\right)=\max _{\Omega} \phi_{f}^{n+1}-\min _{\Omega} \phi_{f}^{n+1}$.

In Eq. 17, the superscript $a$ indicates the result obtained by taking the average of $\phi_{f}, \phi_{o p x}$ and $p$ values over the last two time steps for the corresponding variable. Intuitively, the change in residuals $R e_{i}$ and the jump terms $J_{i}$ which is the difference in the value of the porosity across faces of a cell with respect to the total variation in porosity $N_{h}$ determines the amount of artificial viscosity to be employed for the cell. Implementation and performance of this scheme are discussed in detail in [7, 46]. 
The weak form of the porosity equation with the artificial viscosity term is given by:

$$
\begin{array}{r}
\sum_{k=1}^{K} \int_{D^{k}} \frac{d \phi_{f}}{d t} v d \mathbf{x} \\
=\sum_{k=1}^{K} \int_{D^{k}}\left(\left(\phi_{f}^{n}\right) \boldsymbol{\eta} \cdot \nabla v+\left(\phi_{f}^{n} p^{n}+\Gamma_{o p x}^{n} v\right)\right) d \mathbf{x} \\
-\sum_{e \in \partial D_{i n} \cup \partial \Omega_{d}} \int_{e}\left(\left(\phi_{f}\right)^{*}(\mathbf{n} \cdot \boldsymbol{\eta}) v\right) \\
-\sum_{k=1}^{K} \int_{D^{k}} \nu \nabla \phi_{f}^{n} \cdot \nabla v+\epsilon \sum_{e \in \partial D_{i n} \cup \partial \Omega_{d}} \int_{e}\left\{\nu \nabla v \cdot \mathbf{n}_{e}\right\}\left[\phi_{f}^{n}\right] \\
\sum_{e \in \partial D_{i n} \cup \partial \Omega_{d}} \int_{e}\left\{\nu \nabla \phi_{f}^{n} \cdot \mathbf{n}_{e}\right\}[v]+\sigma \sum_{e \in \partial D_{i n} \cup \partial \Omega_{d}} \int_{e}\left[\phi_{f}^{n}\right][v] \\
+\sum_{e \in \partial \Omega_{d}} \int_{e}\left(\epsilon \nu \nabla v \cdot \mathbf{n}_{e}+\sigma v\right) g_{D} .
\end{array}
$$

Here $\boldsymbol{\eta}=(0,1)$ is the vector field notation that results in the advection of melt in the $\mathrm{z}$ direction in Eq. 1. The diffusion term is discretized using IPDG method similar to the pressure equation. These are given by the last 5 terms on the right hand side in the weak form of the extra diffusion term in Eq. 20. The parameters $\epsilon$ and $\sigma$ are the same as given for the discretization of the pressure equation and $\nu$ is the artificial viscosity added to control the numerical oscillations. This equation also results in two boundary integral terms. The periodic boundary conditions and the Dirichlet boundary conditions need to be taken into account for the diffusion term as well. The Neumann term is taken to be zero as we require only Dirichlet boundary conditions to be satisfied for the original set of governing equations. The values of $\epsilon$ and $\sigma$ were chosen to be 1 for all the simulations. The choice of value for $\alpha$ and consequently $\nu$ for every cell in the mesh is discussed later.

\subsection{Convergence and scaling study}

To verify the convergence of the DG scheme we follow the approach of [35] by taking the same parameter and domain setup as in the previous section, and assuming exact, steady solutions

$\phi_{f_{e}}(x, z, t)=\exp (0.04 z)(1+0.1 \cos (2 \pi x))$,

$\left.p_{e}(x, z, t)=p_{0}+0.1 \cos (2 \pi(x)) z \sin (0.75 \pi z)\right)$,

where $p_{0}$ is the same as the Dirichlet boundary condition specified in Eq. 7. Substituting these exact solutions into Eq. 1 and Eq. 4 yields a residual for each equation that is in turn added back to the equations,

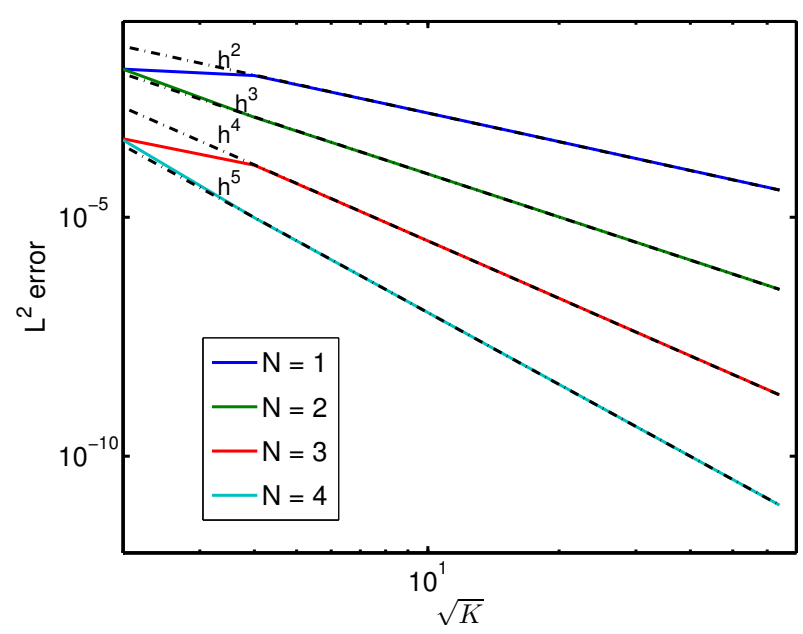

Fig. 1 Convergence study: Error analysis with the manufactured solutions discussed in section 3.3 using Legendre Polynomials $P^{1}, P^{2}, P^{3}$ and $P^{4}$ respectively.
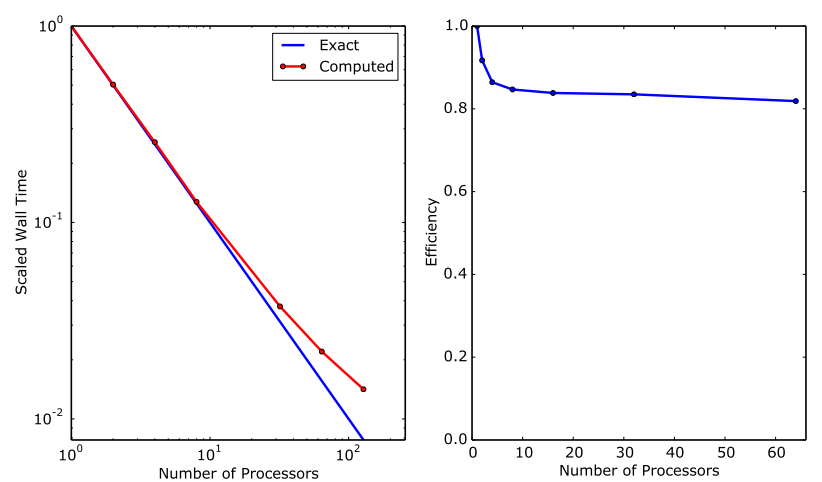

Fig. 2 Strong and weak scaling analysis: Strong scaling using $P^{2}$ polynomials. Weak scaling for a Runge-Kutta time-step with 900 dofs per processor using $P^{3}$ polynomials.

guaranteeing an exact solution as prescribed. We integrate this system to steady state with very small time steps. From general analysis of the DG method in [20] we expect the error of the scheme to diminish as a polynomial function of the size of the element $h$. This is confirmed in Fig. 1, where it is seen that the $L^{2}$-error decays as $h^{(N+1)}$ where $N$ is the degree of polynomial, $K$ are the number of elements and $h$ is the diameter of the cell. Strong scaling and weak scaling studies were done to ensure that the computational time decreases with an increase in the number of processors (strong scaling) and if the load per processor is even (weak scaling). The results in Fig. 2 show that the numerical setup is optimally scalable. Efficiency of weak scaling is defined as the ratio of wall time to complete the work in one processor to the amount of time to complete ' $N$ ' units of the same work with 'N' processors. 


\section{Results from numerical simulations}

Previous studies indicate that dunite channels can be formed through two different instabilities in an upwelling viscously deforming column $[19,28,40]$. To demonstrate the flexibility of DG methods, we consider two benchmark problems in this section, one is the formation of high porosity channels (domain size: $x_{\max }=1, z_{\max }=$ 2 ) and the other is the formation of compaction-dissolution waves $\left(x_{\max }=5.7, z_{\max }=6.8\right)$. The former is used to illustrate the importance of DG methods, local timestepping and isotropic adaptive mesh refinement, while the latter highlights the importance of artificial viscosity, parallelization and anisotropic mesh refinement.

\subsection{Local Time-stepping}

The structure of steady-state high-porosity melt channels and their associated lithologies have been studied in 2D using high order numerical methods $[27,35,36]$. In this section, the efficiency of DG methods with local time-stepping is presented by considering a refined mesh near the channels and a coarse mesh elsewhere. According to the 2D numerical simulations of [36], the imposed boundary condition Eq. 6 results in high porosity channels in the upper part of the simulation domain. Across the high porosity channel, large horizontal gradients in porosity, $\phi_{f}$, and opx fraction, $\phi_{o p x}$, are observed. The numerical method for these results uses $6^{\text {th }}$ order DG elements in the vertical dimension and a Fourier collocation scheme in the horizontal direction. This method consists of a uniformly high order method in the whole domain even though the prominent characteristics of $\phi_{f}$ and $\phi_{o p x}$ are in a small region near the channel boundary. To better and more efficiently resolve these horizontal gradients, we use a non-uniform mesh. The advantage of such a variable mesh is lesser memory usage and improvement in the computational wall time. We can also consider a relatively low order method with this variable mesh to efficiently resolve the dynamics of the problem. In this case, spatial refinement gives the required numerical accuracy achieved through high order methods previously. Therefore, to negate the shortcomings in the numerical methods of $[35,36]$ and $[27]$, we need numerical methods where there are no restrictions on element size and local approximation and DG methods are well suited for problems with variable meshes.

The resulting semi-discrete hyperbolic equations with spatial DG discretization can be integrated using an explicit method as it is simple to implement, requiring only function evaluations of the nonlinear right-hand sides to Eqs. 1 and 2. Also, unlike previous numerical
Table 2 Computational time for the major segments of the numerical scheme for $2^{\text {nd }}$ degree DG polynomials with 6400 cells.

\begin{tabular}{|c|c|c|}
\hline Section & Walltime(s) & \% Total \\
\hline \hline Assemble transport equation & 2.94 & 15.0 \\
\hline Assemble pressure equation & 6.84 & 35.0 \\
\hline Solve transport equation & 0.01 & 0.1 \\
\hline Solve pressure equation & 6.97 & 36.0 \\
\hline
\end{tabular}

studies [40], our model assumes local chemical equilibrium and thus explicit time-stepping is not restricted by a large reaction rate constant. The terms limiting time integration therefore are the upwelling and dissolution terms in Eq. 1 and are typically of comparable magnitude. Time integration can thus be performed with the three-stage, third-order RK method. Each inner RK stage is concluded by discretizing the elliptic equation 4 with the updated value of $\phi_{f}$. However explicit time-stepping methods need to satisfy the Courant-Friedrichs-Lewy(CFL) condition for stability and this is given by:

$\Delta t=C \min _{k}\left\{h_{k}\right\}$,

where $\mathrm{C}$ is a constant depending on the order of the DG polynomials and the stability region of the timestepping algorithm [8]. (Note that the wave speed in the upwelling problems is one.) The drawback of explicit time-stepping methods is that this restriction has to hold globally even if the most refined cells are only a small fraction of the whole domain which is typical of the channel problems in magma dynamics. While the time step is constrained by the hyperbolic equations, it requires us to assemble and solve the elliptic equation after every timestep which accounts for the major computational time in evolving the system of equations over time. This can be seen in Table 2 which gives the time distribution for a domain consisting of $80 \times 80$ cells with $2^{\text {nd }}$ degree DG polynomial elements for a case with $A_{1}=0.1$ in the inflow boundary condition Eq. 6 . Since the pressure equation weakly affects the porosity and opx equations, local time-stepping provides a good alternative to this problem if a method can be developed to efficiently include the constraint imposed on the advection equations because of the pressure equation. While local time-stepping methods have been used for hyperbolic equations before $[6,13,14,17]$, this paper extends the formulation with a constraint for the hyperbolic equations in the form of the pressure equation.

To illustrate the efficiency of local time-stepping methods we consider four cases with refinement levels of $1,2,3$ and 4 respectively at the top of the domain based on the knowledge that there is a strong correlation between the boundary conditions and channel 

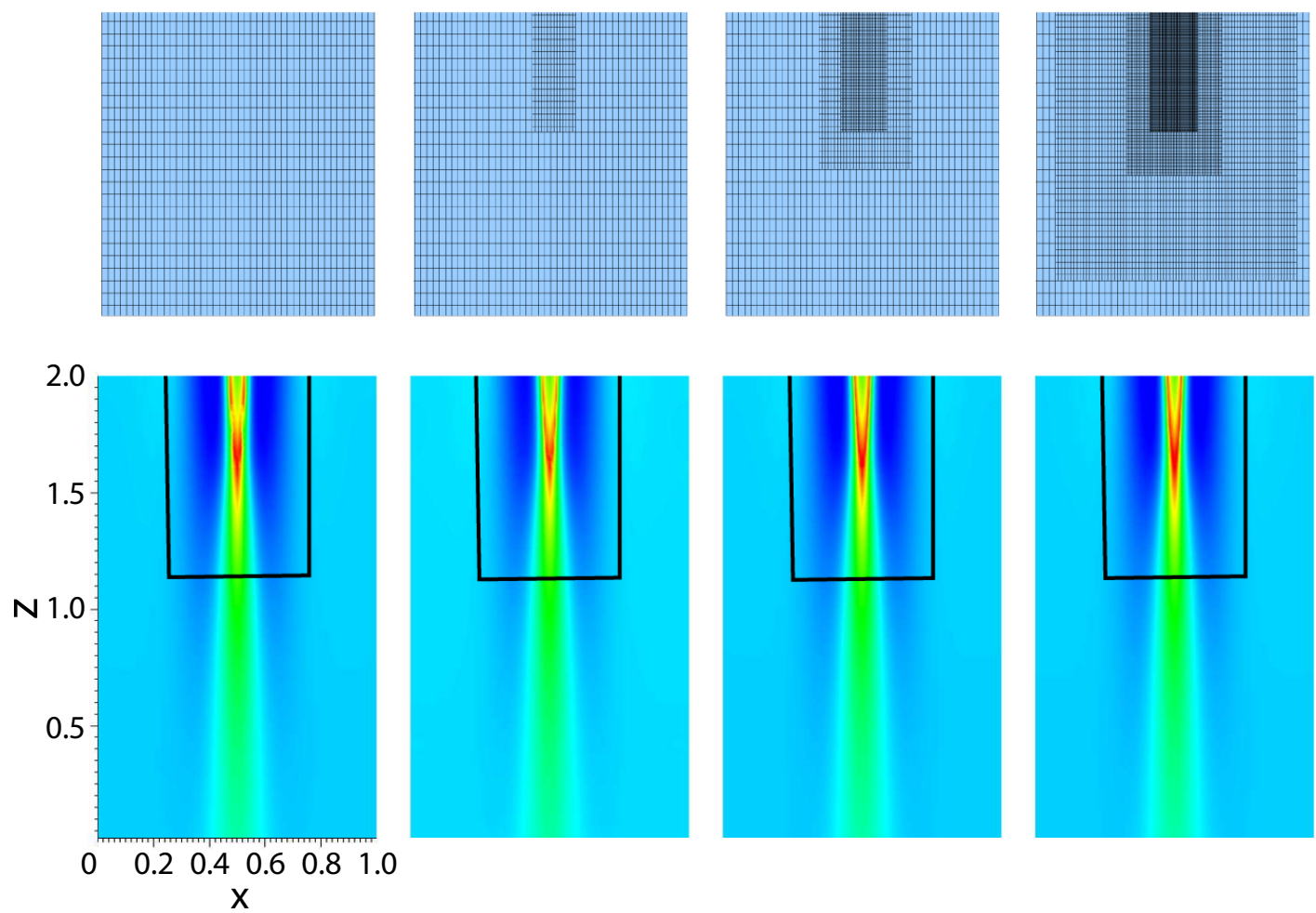

1.8

1.6

1.3

1.1

0.80

Fig. 3 Steady state solutions with sustained Gaussian inflow perturbation for various levels of refinement near the highporosity channel. From left to right, number of levels are 1,2,3 and 4 respectively. Meshes for the regions enclosed by the boxes are shown in the top panel. All the simulations are using $3^{\text {rd }}$ order DG elements with 6400 cells in the coarsest level.

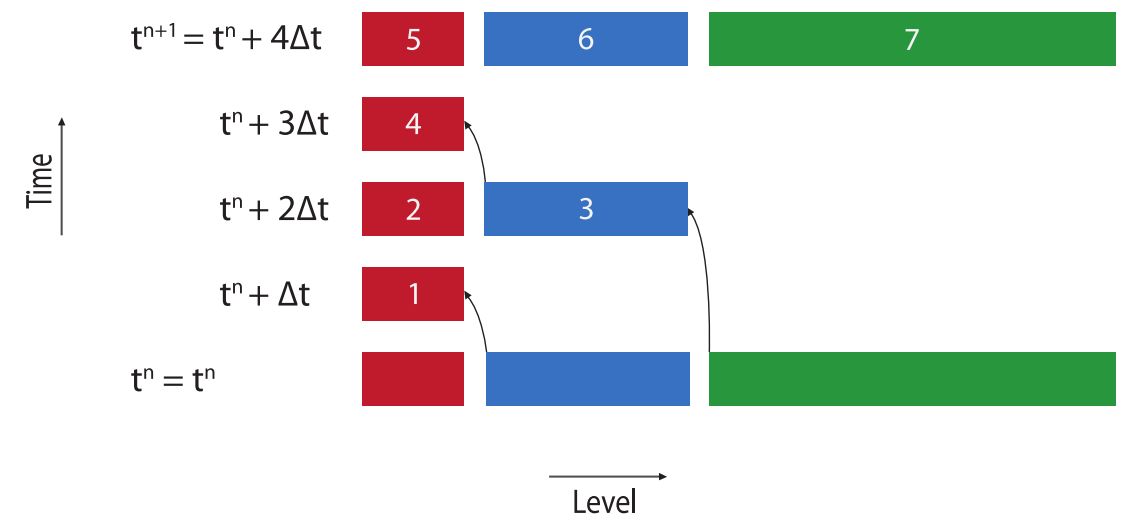

Fig. 4 Local time-stepping schemata for a 3 level mesh. The number indicates the order of evaluation and head of the arrows indicate the timestep at which the coarser neighbor needs to be extrapolated to the half timestep (see text for discussion).

formation [27]. Following [17], let $n_{l}$ define the number of refinement level in the mesh. For example, the coarsest level as shown in the top panel in Fig. 3 has $n_{l}=4$ and the most refined level has $n_{l}=1$. If $\Delta t$ is the maximum stable time step for the most refined level then $\Delta t_{n_{l}}=2^{n_{l}-1} \Delta t$. For local time-stepping methods, time integration is performed at each level $n_{l}$ when the CFL condition at that level needs to be satisfied using 3rd order Adam-Bashforth method (AB3) which is given by:

$\phi_{f}^{n+1}=\phi_{f}^{n}+(\Delta t)_{n_{l}}\left[\frac{23}{12} f_{n}-\frac{16}{12} f_{n-1}+\frac{5}{12} f_{n-2}\right]$,

where $f_{n}$ are the function evaluations from the right hand side in Eq. 11. For the function evaluations, all 
the cells that are in a particular refinement level need only the previous solutions at the time steps corresponding to that level. Solutions are required from the coarser neighbor only for the flux terms and these can be extrapolated using Adam-Bashforth methods by integrating the right hand side for half time step. Like the fundamental derivation of Adam-Bashforth methods, we consider a polynomial $p(t)$ that passes through $f_{n_{l-1}}, f_{n-1_{l-1}}, f_{n-2 l-1}$ for the coarser neighbor as

$p(t)=\sum_{i=0}^{2} f_{n-i_{l-1}} L_{i}(t)$

where $L_{i}(t)$ are the Lagrange polynomials with the interpolation points at the previous time steps that correspond to the three previous stable time steps for that level. Then, the flux terms at half time step required for the flux computations of its refined neighbor can be derived by:

$\phi_{f_{n+\frac{1}{2}}}-\phi_{f_{n}}=\int_{t_{n}}^{t_{n+\frac{1}{2}}} p(s) d s$.

While these methods work as expected for hyperbolic equations, the constraint of the pressure equation which accounts for the major portion of the computational time needs to be solved globally even though the timestep constraint is imposed by the hyperbolic equations. The advantage of using DG methods for the pressure equation is that the solution can be discontinuous across cells. Since the pressure varies slowly with time, the pressure solutions from the previous time steps can be used to extrapolate the solution at the intersection of the coarse and refined cells. Now, to solve the pressure equation locally, the solution from the coarse neighbors from the current level is extrapolated to the half time steps when necessary. These extrapolated values provide the Dirichlet boundary conditions to solve the pressure equation locally. The local time-stepping scheme is illustrated in Fig. 4.

Comparison of total time required to reach steady state for global RK3 and local AB3 methods is given in Table 3 where $n_{L}$ is the total number of levels for the computation. As can be seen from the table, AdamBashforth methods are not efficient for uniform meshes as the CFL condition is 5 times smaller compared to RK3 methods. But as the number of levels increase, where the refined cells are only a small fraction of the total number of cells, the CFL condition is dominated by the number of function evaluations that are required at every time step. Thus, as can be seen in Table 3, when local time-stepping methods are used, the number of function evaluations are limited to the mesh that is needed for the CFL condition to be satisfied. Therefore, the computational wall time decreases as the number of function evaluations on the global mesh at every time step takes a much longer time even though the CFL condition is greater for global Runge-Kutta methods. Adam-Bashforth methods are preferred for local time-stepping methods because these methods result in easier calculation of function values at half time steps as opposed to Runge-Kutta methods. While the results are shown for $2 \mathrm{D}$ channel problems, the percentage gain in computational time would be much more significant for 3D channel problems where the function evaluations and solver time would be significantly more than 2D.

\subsection{Adaptive Mesh Refinement}

While the previous section illustrated the importance of local time-stepping methods by guessing the region of channel formation, it is very difficult to guess the regions of melt migration apriori. DG methods with adaptive mesh refinement can be used if the regions of dunite channel formation is not known. In order to capture the porosity variation at the boundary of the dunite channels, a uniformly refined mesh can be used but it can be computationally prohibitive. As can be seen in the previous case, in Fig. 3, the coarse mesh that was considered does not show channel bifurcation clearly. To generalize the idea from the previous case, adaptive mesh refinement with DG methods provides a good alternative to capture the essential features of melt migration in a computationally efficient manner for a general mesh that is more applicable for realistic problems. Adaptive mesh refinement strategies are employed by refining the mesh where the melt fraction changes rapidly and coarsening the mesh where melt fraction changes slowly. But since the solution at the next time step is not known, the mechanism to define the criterion for regions of refined and coarsened mesh should be made from the values of porosity at the current and previous time steps. This criterion is given by developing an error indicator for porosity since we are interested in the variation of porosity over time. In this case, the error indicator $\eta$ is based on the gradient of porosity as a refined mesh is required to capture the steep gradients and a coarse mesh suffices if gradient is smooth. So for the error indicator, the gradient of porosity is calculated and stored for every cell. Then, the top $20 \%$ of the cells with the highest gradient are marked for refinement and the bottom $20 \%$ of the cells with small gradients are marked for coarsening. In this paper, mesh refinement was done once every 50 timesteps as a trade off between the alignment of mesh and solution variation with the cost of transfering the solution 
Table 3 Wall time with local time-stepping scheme for multilevel meshes.

\begin{tabular}{ccc|cc|ccc}
\hline$n_{L}$ & \multicolumn{2}{c|}{ Assembling } & \multicolumn{2}{c|}{ Solver } & \multicolumn{2}{c|}{ Total Time (s) } & \% Gain \\
\hline \hline & RK3 & AB3 & RK3 & AB3 & RK3 & AB3 & \\
\hline 1 & $2.19 \mathrm{E}+3$ & $3.55 \mathrm{E}+3$ & $1.28 \mathrm{E}+2$ & $2.01 \mathrm{E}+2$ & $2.32 \mathrm{E}+3$ & $3.75 \mathrm{E}+3$ & -61.49 \\
\hline 2 & $4.42 \mathrm{E}+3$ & $4.73 \mathrm{E}+3$ & $2.51 \mathrm{E}+2$ & $3.22 \mathrm{E}+2$ & $4.68 \mathrm{E}+3$ & $5.05 \mathrm{E}+3$ & -8.06 \\
\hline 3 & $1.16 \mathrm{E}+4$ & $8.68 \mathrm{E}+3$ & $1.58 \mathrm{E}+3$ & $1.61 \mathrm{E}+3$ & $1.32 \mathrm{E}+4$ & $1.03 \mathrm{E}+4$ & 22.21 \\
\hline 4 & $4.08 \mathrm{E}+4$ & $3.07 \mathrm{E}+4$ & $7.81 \mathrm{E}+3$ & $3.05 \mathrm{E}+3$ & $4.86 \mathrm{E}+4$ & $3.37 \mathrm{E}+4$ & 30.53 \\
\hline
\end{tabular}
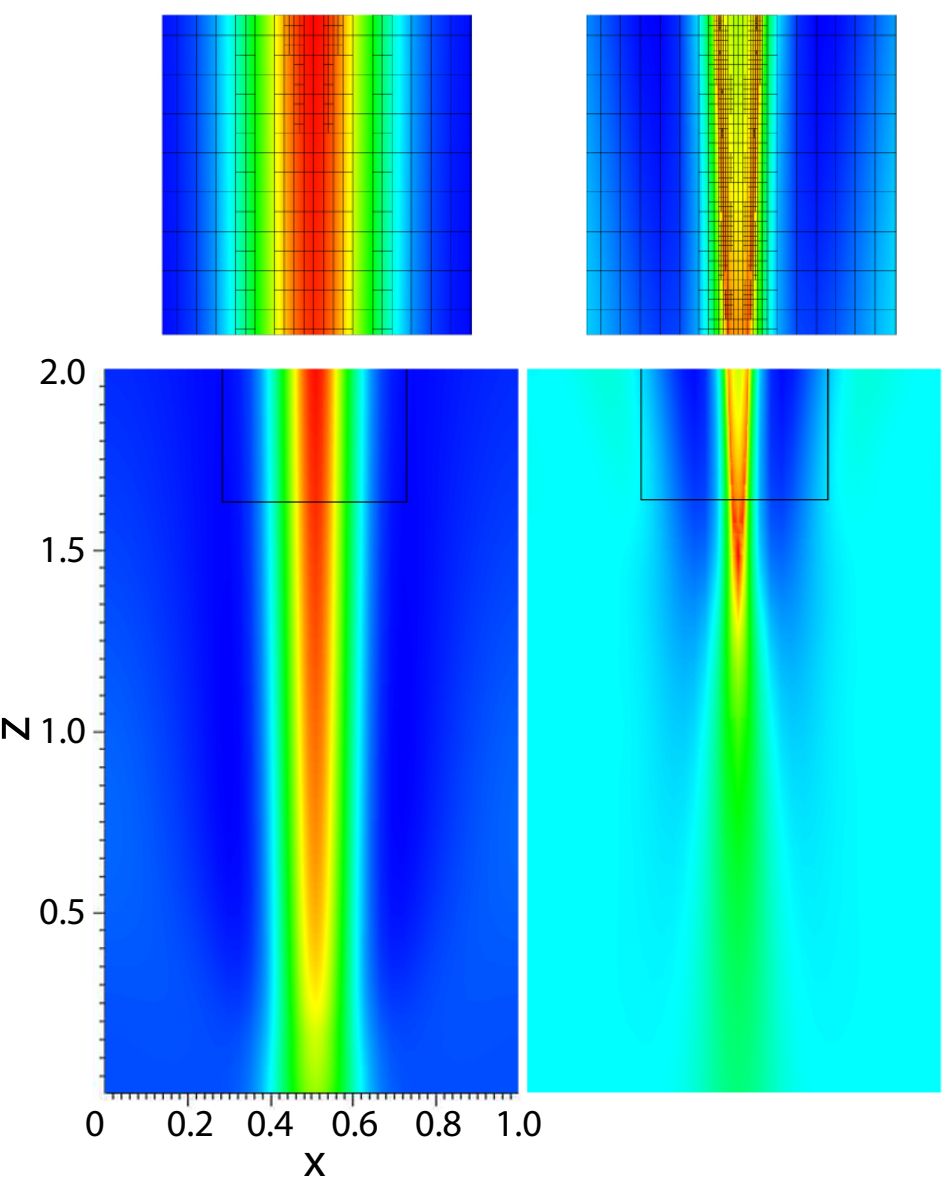
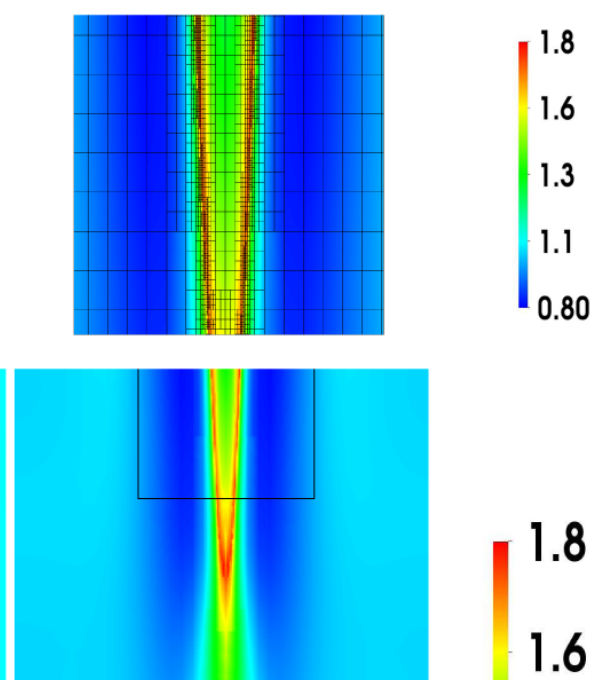

1.3

1.1

0.80

Fig. 5 Bottom: Time variation of fluid fraction with sustained inflow perturbation and adaptive mesh refinement using $4^{t h}$ order DG elements with 5 levels of maximum refinement. From left to right, $t=0.0,1.2,2.4$. Top: Mesh refinement near the channels for the corresponding timesteps. Note: The color scale is for $t=2.4$.

at every time step. As can be seen from Fig. 5, the gradient of porosity as error indicator captures all the essential features of channel formation and the mesh is refined only in the region of dunite channel boundary closer to steady state. The amplitude of the Gaussian perturbation, $A_{1}$ in Eq. 6 , is set to 0.15 in this case. Fig. 6 shows a comparison of the line profile plot of porosity along $z=2$ for uniform and adaptive mesh refinement. We get identical results using both the refinement strategies. The major advantage of using adaptive mesh refinement is that the number of cells and number of degrees of freedom are substantially less to capture the essential features of magma dynamics. The overall computational time also goes down by $40 \%$ with an adaptive mesh for the simulation to run to steady state. 


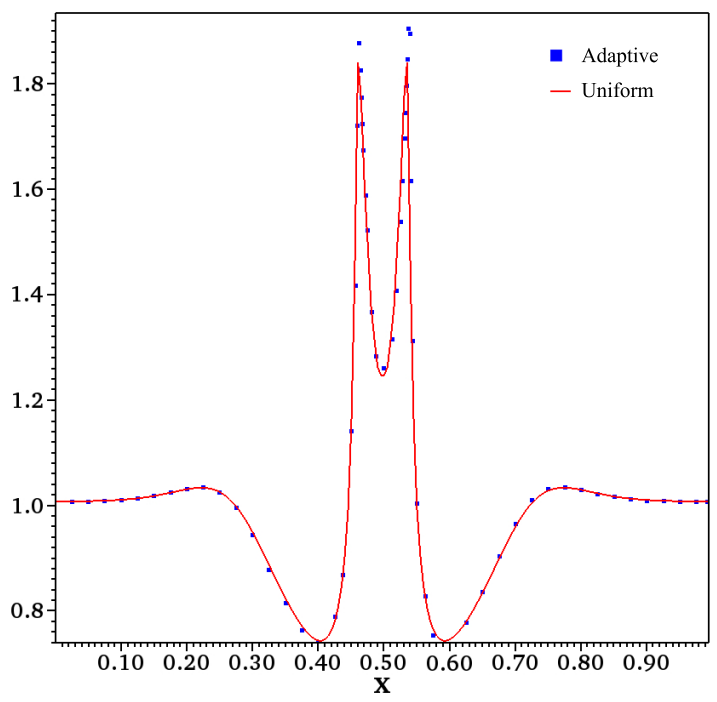

Fig. 6 Line profiles of porosity at $t=2.4$ along $z=2$ using uniform mesh consisting of 10000 cells and adaptive mesh consisting of 4009 cells.

\subsection{Wave regime results with uniform mesh}

Nonlinear interaction among dissolution, compaction, and solid upwelling can also give rise to compactiondissolution waves $[19,28,35,36]$. For this, we consider reactive dissolution regime in a $5.7 \times 6.8$ rectangular domain(measured in compaction length [30]). This domain is an approximation for the upper most part of the upwelling mantle beneath the mid-ocean ridge. Fig. 7 shows the temporal evolution of porosity and opx abundance in the simulation domain. As discussed in Section 3, one of the important considerations for the wave regime computations is the amount of artificial viscosity to control the spurious oscillations. There is a trade off between controlling the numerical oscillations and the accuracy of the solution. If the viscosity per cell is large, then the high porosity channel does not interact with the lower part of the domain and hence presents a very dissipated solution. On the other hand, if the viscosity is less, there are spurious oscillations which give rise to unrealistic solutions. Fig. 8 gives a comparison between the porosity solutions at $t=10$ for viscosity parameters $\alpha=0.3,0.5,0.6$ respectively. As can be seen from the plots, as the viscosity increases, the dissipation is more and the waves are not as strong as the undissipated solution. In the present study, we set the stabilization constant to be $\alpha=0.3$.

Fig. 7 displays spatial and temporal evolutions of porosity (upper panels) and opx fractions in the simulation domain at four selected times. Random initial conditions diffuse out and the random boundary conditions slowly result in well organized compaction-dissolution waves before one solid overturn time $(t=6.7$, the time required for the solid to traverse through the domain once). At the end of the second solid overturn time, compaction-dissolution waves are fully developed at the lower half of the domain while high porosity channels are initiated at the top. The localization of the melt into the channels that are formed in the top half of the domain dissolves the remaining opx and close to the third overturn time $(t=20.4)$ six-well developed opx-free dunite channels are formed. Downward growth of these high porosity channels perturbs the local melt flow field which causes continuous nonlinear interactions between the lower part of the domain and the channels in the upper part eventually leading to a decrease in the wavelength of the porosity waves and an increase in the number of dunite channels to eight by the fourth overturn time after which it reaches a pseudo-steady state. Numerical simulations in the wave regime were compared with those reported in [28]. These results are in qualitative agreement with those reported by [28] for an identical problem (see their Fig. 2) for which a hybrid numerical scheme consisting of DG and a high-order finite difference method was used. In the present study, the domain was discretized into $80 \times 80$ mesh with 4 th order DG methods. These simulations were run in parallel on 128 processors.

\subsection{Wave regime results with anisotropic mesh refinement}

Adaptive mesh refinement can be used in the wave regime context as well. But by noticing that the gradients are sharp only in the $\mathrm{x}$-direction, adaptive mesh refinement can be further improved by making it anisotropic. Based on the error indicators, the mesh is refined only in the $\mathrm{x}$-direction while the mesh is uniform in the $\mathrm{z}$ direction. Every cell marked for refinement is now divided into two cells in the $\mathrm{x}$-direction unlike isotropic mesh refinement where the cell is refined in both the $\mathrm{x}$ and z-direction which results in four refined cells. Fig. 9 shows the formation of dunite channels with three levels of anisotropic mesh refinement in the background. Based on the error indicators for each cell, the top $20 \%$ of the cells are marked for refinement while the bottom $30 \%$ are marked for coarsening. The anisotropic mesh refinement ensures that the mesh is refined only where the channels are formed and coarse everywhere else. These simulations were also run in parallel with 128 processors and the wall time with anisotropic mesh refinement leads to an improvement over uniform mesh solutions by a factor of 2 approximately. 

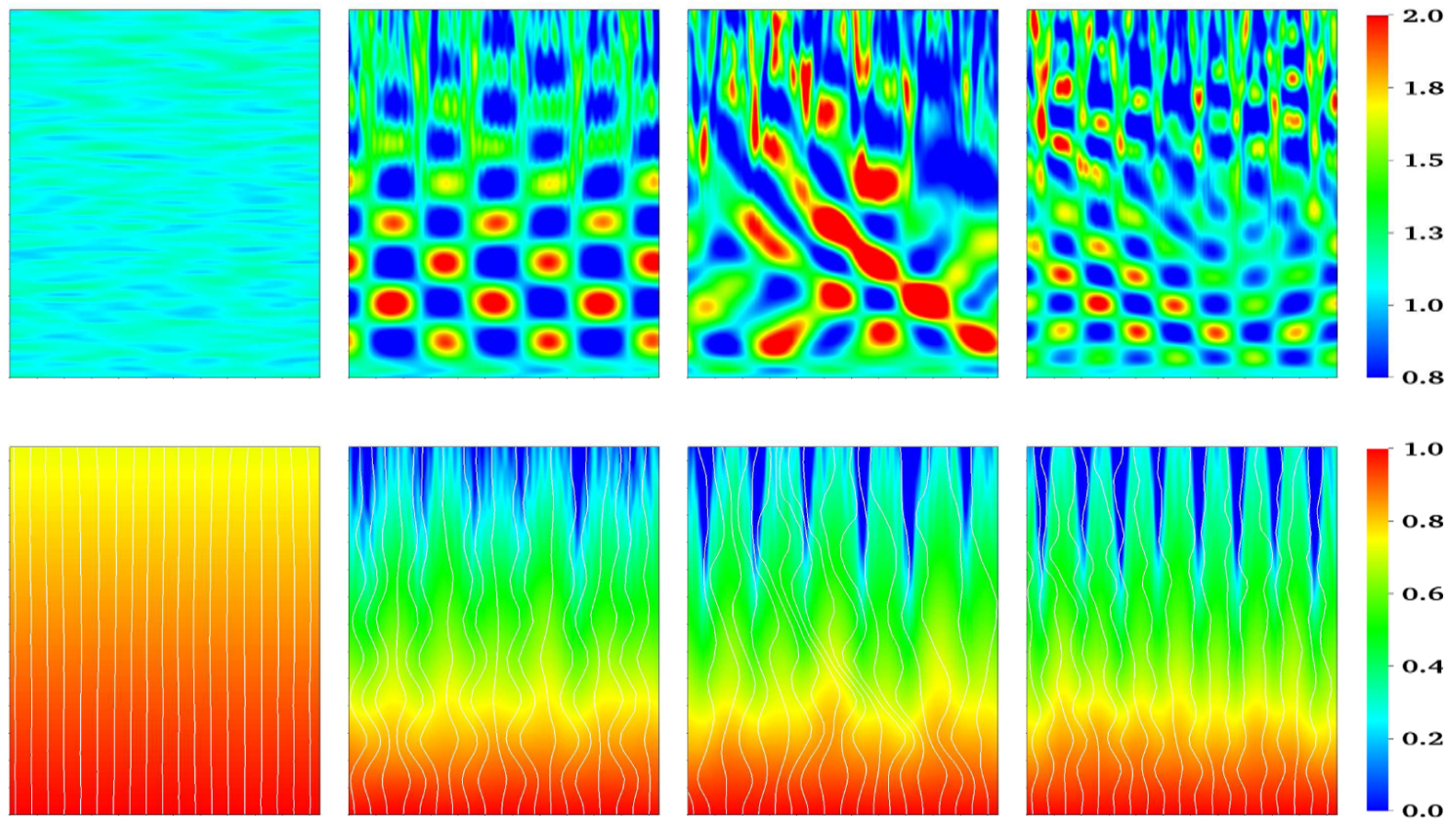

Fig. 7 Time variation of solutions with $4^{\text {th }}$ order DG elements and random inflow perturbation and uniform mesh refinement with 6400 elements. From left to right, $\mathrm{t}=$ 0.0, 6.4, 15.2, 36.2 Top: Fluid fraction. Bottom: Opx fraction.
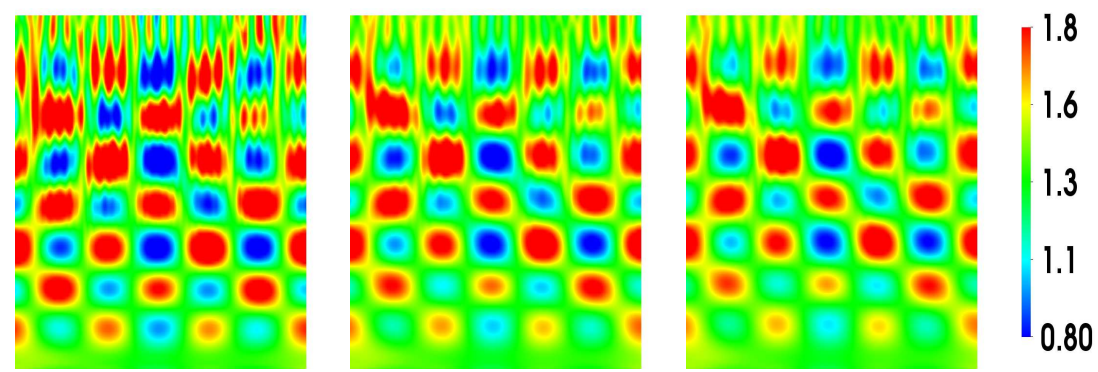

Fig. 8 Porosity at $t=10.0$ with $4^{\text {th }}$ order DG elements with 3600 cells and random inflow perturbation with different stabilization constants $\alpha$. From left to right, $\alpha=0.3,0.5,0.6$.

\section{Summary}

In this paper, discontinuous Galerkin method has been presented for the equations of magma dynamics described by the physical models presented in $[19,35,36]$. This model includes a porosity-dependent bulk viscosity term, a solid upwelling term and a hyperbolic equation to track the fraction of the dissolvable mineral opx. The latter quantity is used such that upon opx exhaustion, the mass transfer rate is taken to be zero. Our numerical implementation consists of an upwinding discontinuous Galerkin method for the advection equations and IPDG method for the elliptic equation. Time integration was done using 3rd order Runge-Kutta method except for local time-stepping methods where 3rd order
Adam-Bashforth methods were used. Artificial viscosity was added to solve the porosity equation in the wave regime. Numerical error estimates match their theoretically expected values and scaling studies show the method's efficiency in parallelization. Assembling and solving the pressure equation was seen as the major bottleneck in solving these problems and local timestepping and adaptive mesh refinement methods have been proposed to significantly decrease the computational time. These methods are well-suited to study high-porosity channels and compaction dissolution waves which are predicted by linear stability analysis. We observe that the compacting boundary layers are present outside high-porosity melt channels. In the event of depleted opx abundance, melt channels bifurcate. The 


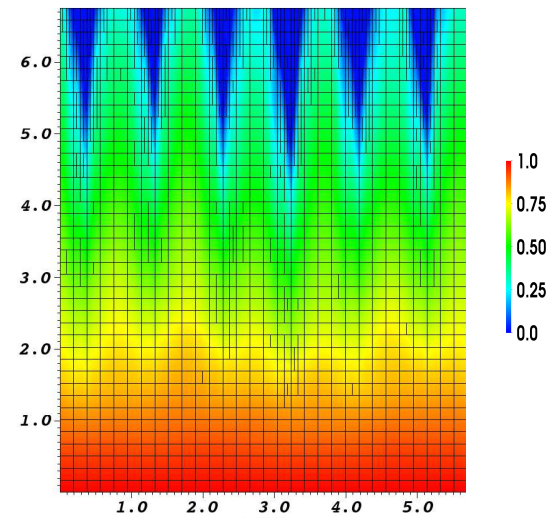

Fig. 9 Spatial vatiations of normalized opx fraction in the simulation domain at $t=15.0$ with anisotropic mesh refinement using 4th order DG elements with a fixed number of 40 elements in the $\mathrm{z}$-direction. The elements in the $\mathrm{x}$-direction are anisotropically refined with 3 levels of maximum refinement.The mesh is refined only in the $\mathrm{x}$-direction.

numerical method developed in this study enables the study of an important but unresolved geological problem regarding the shape or geometry of high-porosity channels in 2D. 2D numerical simulations of high-porosity dunite channel formation in the stable regime suggest a strong correlation between boundary perturbation in porosity at the inflow and dunite channel initiation. This higher than background porosity at the inflow is attributed to higher abundance of fusible materials in the upwelling mantle column [27]. The methods described in this paper can be easily extended to $3 \mathrm{D}$ as well using deal.II which provides a modern interface for dimension independent programming and adaptive mesh refinement $[43,44]$. Geological applications of reactive melt migration in $2 \mathrm{D}$ and $3 \mathrm{D}$ using the numerical methods outlined in this study and those in $[43,44]$ will be presented in a companion study.

\section{Acknowledgments}

We would like to thank the reviewer for the valuable feedback. This work was supported by a grant from the U.S. National Science Foundation (OCE-1156706).

\section{References}

1. Aharonov, E., Whitehead, J., Kelemen P.B., and Spiegelman, M.: Channeling instability of upwelling melt in the mantle. J. Geophys. Res. 100, 433-450 (1995).

2. Arnold, D.N., Brezzi, F., Cockburn, B., and Marini, L.D.: Unified analysis of discontinuous Galerkin methods for elliptic problems. SIAM J. Numer. Anal. 39, 1749-1779 (2002).
3. Bangerth, W., Hartmann, R. , and Kanschat, G.: deal.II: A general-purpose object-oriented finite element library. ACM Trans. Math. Softw. 33(4), 24/1-24/27 (2007).

4. Bercovici, D., Ricard, Y. and Schubert, G.: A two-phase model for compaction and damage 1. General theory. J. Geophys. Res. 106(B5), 8887-8906 (2001).

5. Butler, S.L.: The effects of buoyancy on shear-induced melt bands in a compacting porous medium. Physics of the Earth and Planetary Interiors 173, 51-59 (2009).

6. Castro, C. E., Kaser, M., and Toro, E. F.: Spacetime adaptive numerical methods for geophysical applications. Philosophical Transactions of the Royal Society of London A: Mathematical, Physical and Engineering Sciences 367 4613-4631 (2009).

7. Chueh, C.C., Secanell, M., Bangerth, W., and Djilali, N.: 10. Multi-level adaptive simulation of transient two-phase flow in heterogeneous porous media, Computers and Fluids 39, 1585-1596 (2010).

8. Cockburn, B., and Shu, C.W.: Runge-Kutta discontinuous Galerkin methods for convection-dominated problems. Journal of Scientific Computing 16, 173-261 (2001).

9. Cockburn, B., and Shu, C.W.: The Runge-Kutta local projection P1-discontinuous Galerkin finite element method for scalar conservation laws. Mathematical Model and Numerical Analysis 25, 337-361 (1991).

10. Cockburn, B., and Shu, C.W.: 89. TVB Runge-Kutta local projection discontinuous Galerkin finite element method for conservation laws II: general framework. Mathematics of Computation 52, 411-435 (1989).

11. Cockburn, B., Lin, S.Y., and Shu, C.W.: TVB RungeKutta local projection discontinuous Galerkin finite element method for conservation laws III: one dimensional systems. Journal of Computational Physics 84, 90-113 (1989).

12. Cockburn, B., and Shu, C.W.: The Runge-Kutta local projection discontinuous Galerkin finite element method for conservation laws IV: the multidimensional case. Mathematics of Computation 54, 545-581 (2001).

13. Dumbser, M., Kaser, M., and Toro, E. F.: An arbitrary high-order Discontinuous Galerkin method for elastic waves on unstructured meshes-V. Local time stepping and padaptivity. Geophysical Journal International 171(2) 695717 (2007).

14. Gear, C. and Wells, D.: Multirate linear multistep methods. BIT 24, 484-502 (1984).

15. Ghods A., Arkani-Hamed, J.: Melt migration beneath mid-ocean ridges. Geophys. J. Int. 140, 687-697 (2000).

16. Giraldo F.X., Hesthaven, J.S., and Warburton, T.: Nodal high-order discontinuous Galerkin method for the spherical shallow water equations. J. Comput. Phys. 181, 499-525 (2002).

17. Goedel, N., Schomann, S., Warburton, T., and Clemens, M.: Local timestepping discontinuous Galerkin methods for electromagnetic RF field Problems. EuCAP (2009).

18. Grieco, G., Ferrario, A., Von Quadt, A., Koeppel, V. and Mathez, E. A.: The Zircon-bearing chromitites of the Phlogopite peridotite of Finero (Ivrea Zone, Southern Alps): Evidence and geochronology of a metasomatized mantle slab. J. Petrol. 42, 89-101 (2001).

19. Hesse, M.A., Schiemenz, A. R., Liang, Y. and Parmentier, E. M.: Compaction dissolution waves in an upwelling mantle column. Geophys. J. Int. 187, 1057-1075 (2011).

20. Hesthaven, J.S., Gottlieb, S. and Gottlieb, D.: Spectral methods for time dependent problems. Cambridge University Press 21 (2007).

21. Hesthaven, J.S., and Warburton, T.: Nodal discontinuous Galerkin methods: Algorithms, analysis, and applications. Springer 54 (2008). 
22. Hesthaven, J. S., Warburton, T., Chauviere, C., and Wilcox, L.: High-order discontinuous Galerkin methods for computational electromagnetics and uncertainty quantification. Scientific Computing in Electrical Engineering 403$412(2010)$

23. Katz, R.: Porosity-driven convection and asymmetry beneath mid-ocean ridges. Geochem. Geophys. Geosyst. 11(11), (2010).

24. Kelemen, P. B.: The origin of the land under the sea. Scientific American 300 2, 52-57 (2009).

25. Kelemen,P. B., Hirth, G., Shimizu, N., Spiegelman, M., and Dick, H. J. B.: A review of melt migration processes in the adiabatically upwelling mantle beneath oceanic spreading ridges. Phil. Trans. R. Soc. Lond. 355, 282-318 (1997).

26. Keller, T., May, D.A., and Kaus, B.J.P.: Numerical modelling of magma dynamics coupled to tectonic deformation of lithosphere and crust. Geophys. J. Int. 186, 641-664 (2013).

27. Liang, Y., Schiemenz, A., Hesse, M.A., Parmentier, E.M., and Hesthaven, J.S.: High-porosity channels for melt migration in the mantle: top is the dunite and bottom is the harzburgite and lherzolite. Geophys. Res. Lett. 37 (15) (2010).

28. Liang, Y., Schiemenz, A., and Hesse, M.: Waves, channels, and the preservation of chemical heterogeneities during melt migration in the mantle. Geophys. Res. Lett. 38 (20) (2011).

29. Magde, L.S., and Sparks, D.W.: Three-dimensional mantle upwelling, melt generation, and melt migration beneath segment slow spreading ridges. J. Geophys. Res.: Solid Earth 102, 20571-20583 (2009).

30. McKenzie, D.: The generation and compaction of partially molten rocks. J. Petrol. 25, 713-765 (1984).

31. Reed, W.H. and Hill, T.R.: Triangular mesh methods for the neutron transport equation. Los Alamos Report LAUR-73-479 (1973).

32. Richter, A., Stiller, and J., Grundmann, R.: Stabilized high-order discontinuous Galerkin methods for aeroacoustic Investigations. Computational Fluid Dynamics 77-82 (2009).

33. Riviere, B.: Discontinuous Galerkin methods For solving elliptic And parabolic equations. Frontiers in Applied Mathematics (2008).

34. Saad, Y.: Iterative methods for sparse linear systems (2nd edition). SIAM Publishing (2003) .

35. Schiemenz, A.R., Hesse, M.A., and Hesthaven, J.S.: Modeling magma dynamics with a mixed Fourier collocationdiscontinuous Galerkin method. Commun. Comput. Phys 10(2), 433-452 (2009).

36. Schiemenz, A.R., Liang, Y. and Parmentier, E.M.: A high-order numerical study of reactive dissolution in an upwelling heterogeneous mantle: I. Channelization, channel lithology, and channel geometry. Geophys. J. Int. 186, 641664 (2011).

37. Scott, D. R. and Stevenson, D. J.: Magma ascent by porous flow. J. Geophys. Res. 91, 9283-9296 (1986).

38. Spiegelman, M. and Katz, R.: A semi-Lagrangian CrankNicolson algorithm for the numerical solution of advectiondiffusion problems. Geochem. Geophys. Geosyst. 7, Q04014 (2006).

39. Spiegelman, M. and Kelemen, P.B.: Extreme chemical variability as a consequence of channelized melt transport. Geochem. Geophys. Geosyst. 4, 8615 (2003).

40. Spiegelman, M., Kelemen, P.B. and Aharonov, E.: Causes and consequences of flow organization during melt transport: The reaction infiltration instability in compactible media. J. Geophys. Res. 106, 2061-2077 (2001).
41. Steefel, C., Lasaga, A.: Evolution of dissolution patterns, in Chemical Modeling in Aqueous Systems II. Am. Chem. Soc. 106, 212-225 (1990).

42. Sun, S., and Wheeler, M. F.: Symmetric and nonsymmetric discontinuous Galerkin methods for reactive transport in porous media. SIAM Journal on Numerical Analysis 43(1), 195-219 (2005).

43. Tirupathi, S.: Discontinuous Galerkin methods for magma dynamics. Brown Digital Repository.

44. Tirupathi, S., Hesthaven, J.S., Liang, Y.: Modeling 3D magma dynamics using a discontinuous Galerkin method. Commun. Comput. Phys (to appear) (2015).

45. Van der Vegt, J., and Van der Ven, J.J.W.: Space-time discontinuous Galerkin finite element method with dynamic grid motion for inviscid compressible flows: I. General formulation. Journal of Computational Physics 182(2), 546585 (2002).

46. Zingan, V., Guermond, J. L., Morel, J., and Popov, B.: Implementation of the entropy viscosity method with the discontinuous Galerkin method. Computer Methods in Applied Mechanics and Engineering 253, 479-490 (2012).

\section{Appendix}

Following [36], we consider an effective binary system that consists of an interconnected melt network and a solid matrix comprised both of a soluble mineral, opx and an insoluble mineral, olivine (ol). Let $\Gamma_{o p x}$ be the dissolution rate of opx and $\Gamma_{o l}$ be the precipitation rate of ol in the melt, measured as the volume of mineral dissolved or precipitated per unit volume of the porous medium per unit time. Then $\Gamma_{o l}=-\beta \Gamma_{o p x}$, where $\beta$ is the stoichiometric coefficient for opx dissolution. Mass conservation equations for the melt and the total solid are given by:

$\frac{\partial \phi_{f}}{\partial t}+\nabla \cdot\left(\phi_{f} \mathbf{v}\right)=(1-\beta) \Gamma_{o p x}$,

$\frac{\partial\left(1-\phi_{f}\right)}{\partial t}+\nabla \cdot\left[\left(1-\phi_{f}\right) \mathbf{V}\right]=-(1-\beta) \Gamma_{o p x}$,

where $\mathbf{v}$ and $\mathbf{V}$ are melt and solid velocities, respectively. Conservation of soluble mineral, opx, in the solid matrix is given by

$\frac{\partial \phi_{o p x}}{\partial t}+\nabla \cdot\left(\phi_{o p x} \mathbf{v}\right)=-\Gamma_{o p x}$,

The volume fraction of olivine is derived from the volume fractions of $\phi_{o p x}$ and $\phi_{f}$ as

$\phi_{o l}=1-\phi_{o p x}-\phi_{f}$,

Eqs. 23 - 25 are derived using the Boussinesq approximation i.e., that the densities of the melt and the solid are the same and that the velocities of opx and ol are the same as the bulk solid velocity. Neglecting diffusion and dispersion in the melt and assuming constant and 
uniform mineral compositions, mass conservation equation for the independent component in the melt for the effective binary system is given by :

$\frac{\partial\left(\phi_{f} c_{f}\right)}{\partial t}+\nabla \cdot\left(\phi_{f} \mathbf{v} c_{f}\right)=\left(c_{o p x}^{0}-\beta c_{o l}^{0}\right) \Gamma_{o p x}$,

where $c_{f}, c_{o p x}^{0}$ and $c_{o l}^{0}$ are concentrations of the independent component in the melt, opx and ol, respectively. Mass transfer can be modelled by the first-order kinetic approximation $[1,40,41]$

$\Gamma_{o p x}=\alpha_{r} \phi_{f} \phi_{o p x}\left(c_{f}^{e q}(\mathbf{x})-c_{f}, \quad 0<\alpha_{r}<\infty\right.$,

where coefficient of proportionality $\alpha_{r}$ is a rate constant. For the effective binary system considered in this study, the equilibrium concentration, $c_{f}^{e q}$ varies only as a function of the vertical coordinate if the effect of temperature is small compared to that of pressure.

$c_{f}^{e q}(\mathbf{x})=c_{f}^{e q}(z)=c_{f}^{0}+\Delta c F(z / L)$,

At the lower boundary of our domain the solubility takes the value $c_{f}=c_{f}^{0}$, and $\Delta c$ is the change in solubility over a compaction length $L$. In the limit $\alpha_{r} \rightarrow \infty$, concentration $C_{f}$ tends to equilibrium value and the dissolution rate is obtained by combining Eqs. 23 and 27 with $c_{f}=c_{f}^{e q}$ :

$\Gamma_{o p x}=\frac{\phi_{f}\left(\mathbf{v} \cdot \mathbf{n}_{\mathbf{z}}\right) \partial c_{f}^{e q} / \partial z}{c_{o p x}^{0}-\beta c_{o l}^{0}-(1-\beta) c_{f}^{e q}} \mathcal{I}_{o p x}$,

where $\mathbf{n}_{z}$ is the unit vector in the vertical dimension and where the indicator function $\mathcal{I}_{o p x}$ returns 1 if $\phi_{o p x}$ is positive and zero otherwise. In the equilibrium limit, the opx dissolution rate is proportional to the vertical melt flux and solubility gradient and is independent of opx abundance.

Darcy's law, relating relative melt flux to gradients of an effective pressure and melt buoyancy is used to determine the velocities, $\mathbf{v}$ and $\mathbf{V}$,

$\phi_{f}(\mathbf{v}-\mathbf{V})=-\frac{\kappa_{\phi}}{\mu}\left[\nabla p-\Delta \rho g \boldsymbol{n}_{z}\right]$

where $\kappa_{\phi}$ is the permeability, $\mu$ is the viscosity of the melt, $p=p_{f}-p_{s}$ is the pressure difference between the melt $\left(p_{f}\right)$ and the solid $\left(p_{s}\right), \Delta \rho=\rho_{s}-\rho_{f}$ is the density difference between the solid $\left(\rho_{s}\right)$ and the melt $\left(\rho_{f}\right)$, $g$ is the acceleration due to gravity. The permeability depends on porosity through the power-law relation,

$\kappa_{\phi}=\kappa_{0}\left(\frac{\phi_{f}}{\phi_{f}^{0}}\right)^{n}$

where $\kappa_{0}$ is the permeability at the reference melt fraction $\phi_{f}^{0}$ and $n=3$ in the present study. The pressure difference between the melt and the solid is related to the compaction rate of the matrix $(\nabla \cdot \boldsymbol{V})$ through the bulk velocity $\xi$,

$p=p_{f}-p_{s}=\xi \nabla \cdot \mathbf{V}$,

$\xi=\frac{\eta}{\phi_{f}}$

where $\eta$ is the shear viscosity of the solid matrix. The effective pressure can be solved from a Helmholtz equation obtained by combining Eqs. 23 and 24 and the divergence of Eq. 31,

$$
\nabla \cdot\left[\frac{\kappa_{\phi}}{\mu}\left(\nabla p-\Delta \rho g \boldsymbol{n}_{z}\right)\right]=\frac{\phi_{f} p}{\eta} .
$$

Eq. 24 can also be written in terms of the effective pressure,

$\frac{\partial \phi_{f}}{\partial t}+\mathbf{V} \cdot \nabla \phi_{f}=\left(1-\phi_{f}\right) \frac{\phi_{f} p}{\eta}+(1-\beta) \Gamma_{o p x}$,

To derive the working model used in this study, we nondimensionalize Eqs. 23 - 36 with the following substitutions:

$t=t^{0} t^{\prime}, \quad \boldsymbol{x}=L \boldsymbol{x}^{\prime}, \quad \phi_{f}=\phi_{f}^{0} \phi_{f}^{\prime}, \quad \phi_{o p x}=\phi_{o p x}^{0} \phi_{o p x}^{\prime}$,

$c_{f}=c_{f}^{0}+\Delta c c_{f}^{\prime}, \quad p=p^{0} p^{\prime}, \quad \boldsymbol{v}=w_{0} \boldsymbol{v}^{\prime}$,

$$
\boldsymbol{V}=W_{0} \boldsymbol{n}_{z}+\Delta V \boldsymbol{V}^{\prime}
$$

where $W_{0}$ is the solid upwelling rate, $w_{0}$ is the inflow melt velocity and $p^{0}$ is the pressure scale. The time, length, pressure and velocity scales are

$t^{0}=\frac{L}{W^{0}}, \quad L=\sqrt{\frac{\kappa_{0} \eta}{\mu \phi_{f}^{0}}}, \quad p^{0}=\frac{\eta W_{0}}{L}$,

$w_{0}=\frac{\kappa_{0} \Delta \rho g}{\phi_{f}^{0} \mu}, \quad \Delta V=\frac{\phi_{f}^{0} w_{0}(1-\beta) \Delta c}{c_{o p x}^{0}-\beta c_{o l}^{0}-(1-\beta) c_{f}^{0}}$,

where $L$ is the compaction length [30], $t^{0}$ is the time needed for a matrix panel to advect a compaction length via solid upwelling. Substituting Eqs. 37 and 38 into Eqs. 25, 27, 31, 35 and 36 and after dropping the primes for clarity, we have the dimensionless equations.

$$
\begin{aligned}
& \frac{\partial \phi_{f}}{\partial t}=-\frac{\partial \phi_{f}}{\partial z}+\phi_{f} p+\delta \Gamma_{o p x} \\
& \frac{\partial \phi_{o p x}}{\partial t}=-\frac{\partial \phi_{o p x}}{\partial z}-\frac{\phi_{f}^{0}}{\phi_{o p x}^{0}} \frac{\delta}{(1-\beta)} \Gamma_{o p x} \\
& -\nabla \cdot\left[\phi_{f}^{3} \nabla p\right]+\phi_{f} p=-R \frac{\partial \phi_{f}^{3}}{\partial z} \\
& \phi_{f} \mathbf{v}=R^{-1} \phi \mathbf{n}_{z}-\phi_{f}^{3}\left(R^{-1} \nabla p-\mathbf{n}_{z}\right)
\end{aligned}
$$


$\Gamma_{o p x}=R \frac{\phi_{f}\left(\mathbf{v} \cdot \mathbf{n}_{z}\right)}{1-\delta z} \mathcal{I}_{o p x}$

Eqs. 39 - 42 have three dimensionless parameters,

$\frac{\phi_{f}^{0}}{\phi_{o p x}^{0}}, \delta=(1-\beta) \frac{\Delta c}{\hat{c}}, R=\frac{w_{0}}{W_{0}}$,

where $\hat{c}=c_{o p x}^{0}-c_{o l}^{0}-(1-) c_{f}^{0}$ is a characteristic coefficient. 